\title{
Research on Vibration Characteristics of Piezoelectric Ceramic Atomizer Based on ANSYS
}

\author{
Yan Chen ${ }^{1, *}$, Chunyan $\mathrm{Ma}^{1}$, Zaihe Shen ${ }^{2}$ and Rui Chen ${ }^{1}$ \\ ${ }^{1}$ College of Electrical and Power Engineering, Taiyuan University of Technology, Taiyuan 030024, Shanxi Province, China; \\ ${ }^{2}$ State Grid Hebei Electric Power Company, Ningjin County Power Supply Company, Xingtai 055550, Heibei Province, China.
}

\begin{abstract}
In the design and application of ultrasonic atomizer, the resonant frequency and vibration mode of piezoelectric transducer have an effect on the working state and atomization effect of the atomizer. In order to deeply study the piezoelectric ceramic ultrasonic atomizer Vibration characteristics, piezoelectric coupling simulation analysis was performed by ANSYS software, multi-order vibration mode of piezoelectric ceramic atomization sheet obtained by modal analysis method, combined with harmonic response analysis to obtain resonant frequency of piezoelectric ceramic ultrasonic atomization sheet, and analysis the influence of the main size of the atomized sheet on the vibration mode. According to theoretical analysis and experimental tests, the effectiveness of the finite element analysis can be verified, and then provide a theoretical basis for the study of ultrasonic atomizers.
\end{abstract}

\section{Introduction}

Ultrasonic atomization technology is widely used in agricultural aerosol cultivation, medical inhalation therapy, air humidification and many other fields because of its good atomization effect, uniform fog and small particle size. The ultrasonic atomizer generates highfrequency pressure waves by using the piezoelectric effect. The liquid medium is subjected to high-frequency vibration by the influence of ultrasonic waves, and alternately stretches and contracts on the surface of the liquid to form a surface tension wave. When the vibration reaches a certain intensity, the liquid is in the air. In the process of dispersion, numerous tiny droplets are formed to achieve atomization [1].

The core of the ultrasonic atomizer is a piezoelectric transducer, which is bonded by a piezoelectric vibrator ring and a metal cover [2]. With the inverse piezoelectric effect, the piezoelectric transducer generates highfrequency oscillation deformation when a high-frequency electric field is applied. When the resonant frequency of the piezoelectric crystal is equal to the frequency of the applied electric field, the resonance reaches a mechanical resonance state, which in turn produces high frequency vibration [3]. At this time, the electromechanical energy conversion efficiency is the highest, and the atomizer has the highest working efficiency. Therefore, studying the resonant frequency of the ultrasonic atomizer is important for the atomization effect. The finite element analysis method is a numerical analysis method widely used in engineering practice [4]. This paper will use ANSYS software to simulate and analyse the piezoelectric ceramic ultrasonic atomization sheet, by comparing with the Theoretical value, the effectiveness and accuracy of ultrasonic nebulizers can be studied by using the finite element analysis.

\section{Vibration Simulation Analysis of Piezoelectric Ceramic Atomized Sheet}

The finite element analysis method is also called numerical analysis method. The basic principle is based on the variational principle and the split interpolation. The continuous structure is decomposed into a finite number of tiny elements, the node interpolation function is constructed, the equations are established, and the solution is solved by computer. Finally, observation graphs or tables are used to analyse the results in Postprocessing links [5].

\subsection{ANSYS Modeling and Meshing}

The piezoelectric ceramic ultrasonic atomizing sheet is formed by bonding a ring-shaped piezoelectric ceramic sheet and a circular metal plate. The schematic structure of the piezoelectric ceramic ultrasonic atomizing sheet is shown in Figure 1. The piezoelectric ceramic ultrasonic wave with a theoretical resonant frequency of $160 \mathrm{kHz}$ is obtained. The atomization sheet structure and material parameters are shown in Table 1.

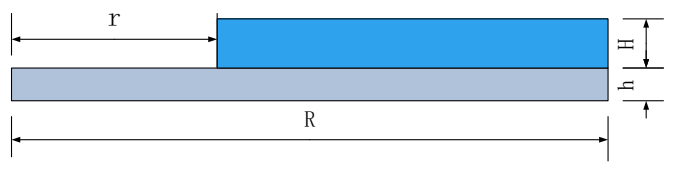

Fig. 1. The structure of piezoelectric ceramic ultrasonic atomizing sheet.

\footnotetext{
* Corresponding author: chenyanlxq@163.com
} 
Table 1. Piezoelectric transducer sheet parameter.

\begin{tabular}{|c|c|c|}
\hline Parameter & SUS304 & PZT4 \\
\hline Elastic Modulus (Gpa) & 206 & - \\
\hline Poisson's ratio & 0.3 & - \\
\hline Density $\left(\mathrm{kg}^{\wedge} \mathrm{m}^{3}\right)$ & 7930 & 7500 \\
\hline Outer diameter/R (mm) & 5 & 5 \\
\hline Inner diameter/r (mm) & - & 2.5 \\
\hline Thickness/H (mm) & 0.12 & 0.6 \\
\hline
\end{tabular}

The ultrasonic atomized sheet was modeled in the pretreatment section. The piezoelectric ceramic material (PZT4) was selected from SOLID5 structural unit, and the metal wafer material was selected as SOLID45 structural unit [6]. The density, Poisson's ratio and dielectric properties of the two materials were respectively set. Parameters such as electrical constant, piezoelectric coefficient and elastic modulus.

Due to the good symmetry of the model, one-quarter modeling can be used to reduce the number of grids and save computing time. The mesh sweep is performed by the VSWEEP method. The finite element model summary is viewed through the List. The number of nodes divided by the model is 1600 , and the number of meshes is 1044 . Applying boundary conditions to the model structure, since the degrees of freedom of each unit of the model are mutually coupled, it is necessary to couple the upper and lower surface nodes and apply full boundary displacement constraints [7]. The effect of applying boundary constraints and degrees of freedom coupling is shown in Figure 2.

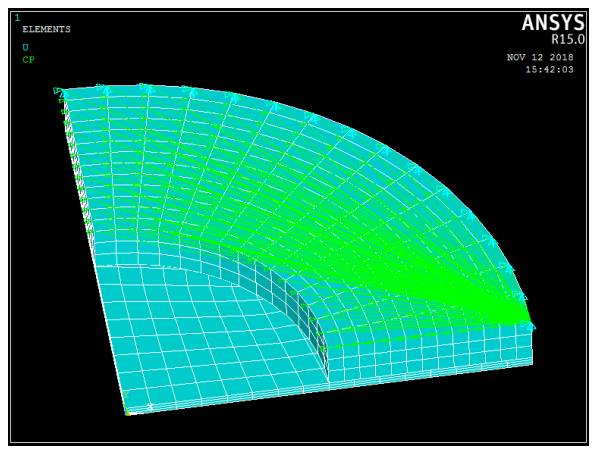

Fig. 2. The figure of applying boundary constraints and degrees of freedom coupling effect.

\subsection{Modal analysis}

Modal analysis is used to analyse the vibration characteristics of the structure in order to determine the natural frequency and mode shape of the structure. It can visually represent the vibration of the structure under the constraints. As one of the foundations of the dynamic analysis, it determines Whether the system structure can achieve the optimization of power conversion [8].

In the analysis and solving module, the analysis type is defined as modal analysis, which is solved by Block Lanczos method, and is set in the frequency range of 20 $\mathrm{kHz}$ to $260 \mathrm{kHz}$ to extract the vibration mode of the 16th order. The natural frequency table of the 16th order is shown in Table 2.

Table 2. 16 order natural frequency table.

\begin{tabular}{|c|c|c|c|c|}
\hline $\begin{array}{c}\text { Modal } \\
\text { order }\end{array}$ & $\mathbf{1}$ & $\mathbf{2}$ & $\mathbf{3}$ & $\mathbf{4}$ \\
\hline $\begin{array}{c}\text { Natural } \\
\text { frequency } \\
\text { (kHz) }\end{array}$ & 30.069 & 38.934 & 69.347 & 69.984 \\
\hline $\begin{array}{c}\text { Modal } \\
\text { order }\end{array}$ & $\mathbf{5}$ & $\mathbf{6}$ & $\mathbf{7}$ & $\mathbf{8}$ \\
\hline $\begin{array}{c}\text { Natural } \\
\text { frequency } \\
\text { (kHz) }\end{array}$ & 83.544 & 95.700 & 101.364 & 120.043 \\
\hline $\begin{array}{c}\text { Modal } \\
\text { order }\end{array}$ & $\mathbf{9}$ & $\mathbf{1 0}$ & $\mathbf{1 1}$ & $\mathbf{1 2}$ \\
\hline $\begin{array}{c}\text { Natural } \\
\text { frequency } \\
\text { (kHz) }\end{array}$ & 151.609 & 165.682 & 178.699 & 181.202 \\
\hline $\begin{array}{c}\text { Modal } \\
\text { order }\end{array}$ & $\mathbf{1 3}$ & $\mathbf{1 4}$ & $\mathbf{1 5}$ & $\mathbf{1 6}$ \\
\hline $\begin{array}{c}\text { Natural } \\
\text { frequency } \\
\text { (kHz) }\end{array}$ & 186.395 & 234.910 & 255.656 & 257.246 \\
\hline
\end{tabular}

Then use the general post-processor to view the vibration deformation of the atomized sheet, and select a representative 4 th and 10 th order vibration mode diagram as shown in Figure 3.

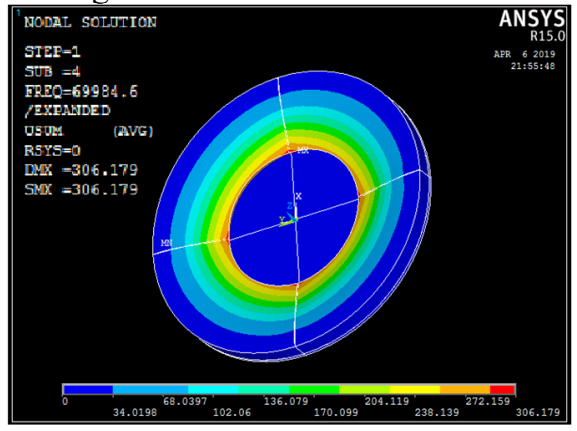

(a) 4th order $(69.984 \mathrm{kHz})$

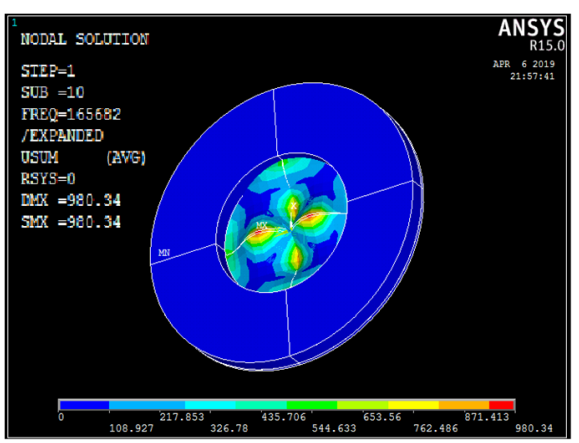

(b) 10 th order $(165.682 \mathrm{kHz})$

Fig. 3. Vibration deformation diagram of atomized sheet 
It can be seen from Fig. 3 that there is a peak in the central region of the 10th-order atomizer, and the vibration is severe. If there is sufficient amplitude and vibration speed, the atomizer can be atomized under the vibration mode, and the fourth-order vibration mode is unavailable. Vibration mode, due to the actual operation of the atomizing sheet, the metal substrate boundary needs external force clamping. If the piezoelectric ceramic ring has severe vibration, the outer boundary vibration of the metal substrate will be more severe, it is difficult to find reasonable fixing measures, and Seriously affect the service life of the atomized sheet. Therefore, the principle of selecting a suitable mode shape is that the vibration at the center of the atomizing sheet is severe, and the vibration amplitude of the piezoelectric ceramic ring is weak to ensure the service life of the atomizing sheet.

\subsection{Harmonic response analysis}

The harmonic response analysis is to analyse the forced vibration of the linear structure and the steady-state dynamic behavior with time, and give the response curve of the displacement of the structure with the frequency, so as to obtain the response peak and the structural stress under the peak structure [9]. Through the harmonic response analysis, using the FULL method and selecting the sparse matrix solver, the relationship between the response peak and the frequency can be obtained. For the atomizers of different sizes, the response peak value can be obtained under the harmonic response condition, and the atomizer can be optimized.

The voltage peak across the piezoelectric ceramic is set to $20 \mathrm{~V}, 20 \mathrm{kHz} \sim 260 \mathrm{kHz}$, and the harmonic response analysis is performed at $100 \mathrm{~Hz}$. After the ANSYS operation is completed, select $0.2 \mathrm{~mm}$ from the center point to check the harmonic response. The vibration amplitude at the center point varies with frequency as shown in Figure 4.

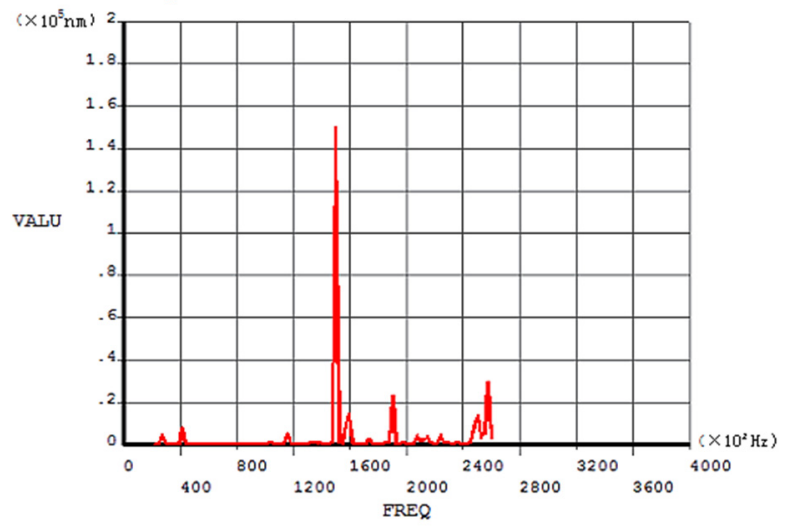

Fig. 4. Vibration amplitude at the center point as a function of frequency

As can be seen from the curve in the figure, at the frequency of $158.927 \mathrm{kHz}$, the maximum displacement of the atomizing sheet at the center position is $151.9 \mathrm{um}$, and the atomizing sheet has the largest vibration amplitude. The theoretical atomization effect is the best. The generalpurpose post-processor is used to view the vibration deformation of the atomization piece at $158.927 \mathrm{kHz}$ into a usable vibration mode, so $158.927 \mathrm{kHz}$ can be used as the resonance frequency.

\section{Effectiveness of finite element analysis of ultrasonic atomizer}

The piezoelectric ceramic ultrasonic atomizing sheets with resonant frequency design values of $108 \mathrm{kHz}, 113$ $\mathrm{kHz}$ and $160 \mathrm{kHz}$ were modeled and analysed to verify the effectiveness of ANSYS in the vibration analysis of piezoelectric ceramic ultrasonic atomizing sheets. Finally, the resonant frequency simulation values were obtained. The simulation value and design value pair are shown in Table 3.

Table 3. Comparison of simulated values and design values of different atomizers.

\begin{tabular}{|c|c|c|c|}
\hline Kind & $\mathbf{1}$ & $\mathbf{2}$ & $\mathbf{3}$ \\
\hline Inner diameter (mm) & 2.5 & 3.9 & 4.0 \\
\hline Outer diameter (mm) & 5 & 8 & 7.9 \\
\hline $\begin{array}{c}\text { Ceramic thickness } \\
\text { (mm) }\end{array}$ & 0.6 & 0.6 & 0.6 \\
\hline $\begin{array}{c}\text { Metal wafer thickness } \\
\text { (mm) }\end{array}$ & 0.12 & 0.17 & 0.16 \\
\hline $\begin{array}{c}\text { Design value (mm) } \\
\text { Simulation value } \\
\text { (mm) }\end{array}$ & 160 & 113 & 108 \\
\hline Amplitude (10²um) & 1.519 & 1.825 & 1.693 \\
\hline
\end{tabular}

It can be seen from Table 3 that the simulation values of the resonant frequency of different atomizers and the design value are within $2 \mathrm{kHz}$, which is sufficient to prove the effectiveness of finite element analysis in determining the resonant frequency of piezoelectric ceramic ultrasonic atomizer.

In order to verify the atomization effect of different atomized sheets, the piezoelectric ceramic ultrasonic atomized sheets of $108 \mathrm{kHz}, 113 \mathrm{kHz}$ and $160 \mathrm{kHz}$ were subjected to atomization experiments under the same liquid composition, with black as the background, and connected by high speed camera at the same angle and distance. In the shooting mode, the atomizer starts to work and the mist does not settle, and the atomization effect of the atomizer can be accurately determined. The atomization effect diagram of different atomized sheets is shown in Figure 5. Among them, the $113 \mathrm{kHz}$ atomization sheet has the best atomization effect, the nutrient solution particle group is the largest, and after ANSYS finite element simulation analysis, the $113 \mathrm{kHz}$ atomization sheet has a center point amplitude of $182.5 \mathrm{um}$ at the resonance frequency, and the $108 \mathrm{kHz}$ and $160 \mathrm{kHz}$ atomization sheets The amplitudes are 151.9um and 169.3um, respectively, indicating that the greater the 
vibration displacement at the center point, the higher the amount of fog, and the better the atomization effect.

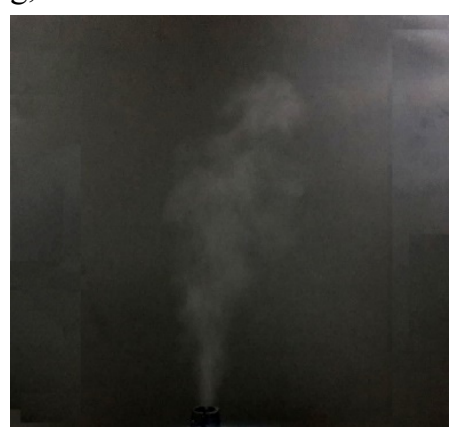

(a) $108 \mathrm{kHz}$

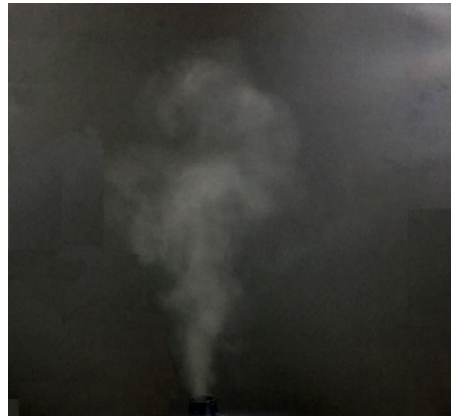

(b) $113 \mathrm{kHz}$

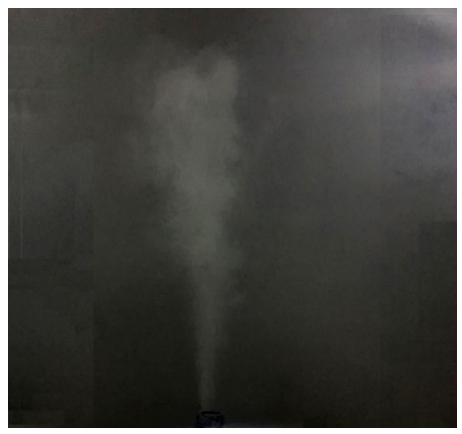

(c) $160 \mathrm{kHz}$

Fig. 5. Atomization effect diagram of different atomized sheets

\section{Influence of different parameter sizes on the vibration of the atomized sheet}

In order to investigate the influence of various main parameters of piezoelectric ceramic atomizing sheet on the resonant frequency and the amplitude of the center point, this paper changes the geometric parameters by modifying the APDL code in ANSYS software, respectively, and the inner diameter and outer diameter of the ultrasonic atomizing sheet with a design value of 113 $\mathrm{kHz}$. The diameter, the thickness of the metal sheet and the thickness of the piezoelectric ceramic ring are modified by different amplitudes for simulation analysis, and the relationship between the different structural parameters and the resonant frequency of the atomizing sheet and the amplitude of the center point can be obtained as shown in Figure 6.

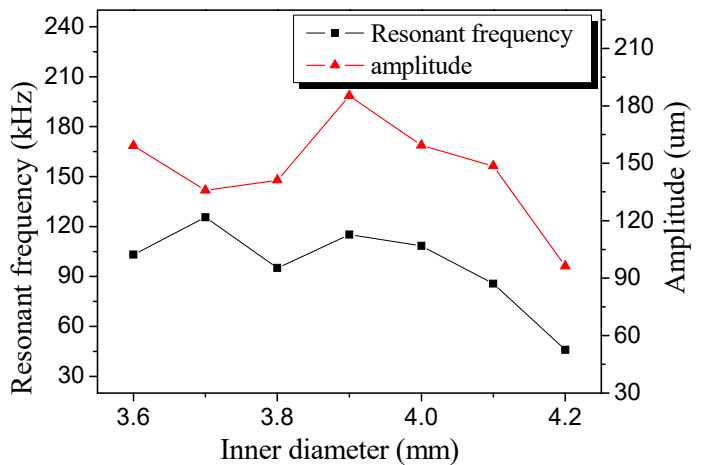

(a) Relationship between inner diameter and resonant frequency and amplitude

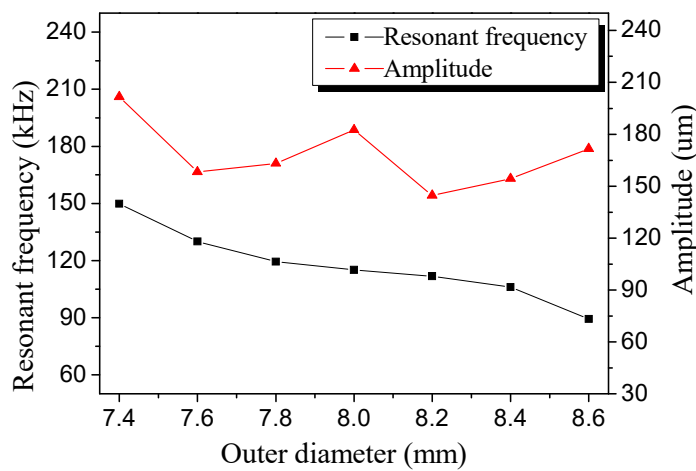

(b) Relationship between outer diameter and resonant frequency and amplitude

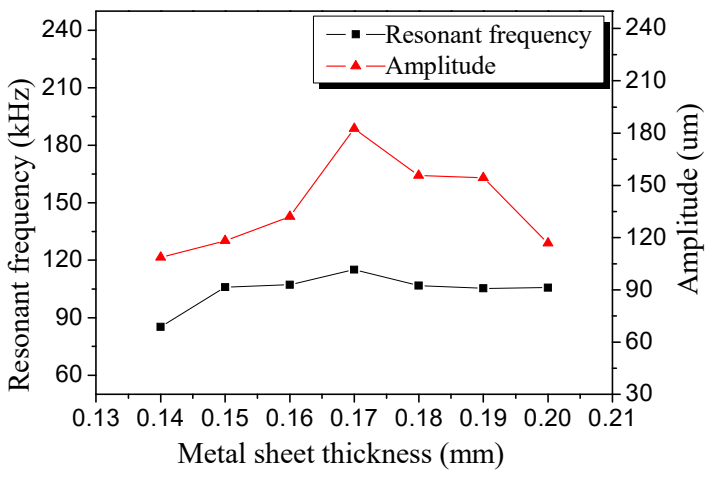

(c) The relationship between the thickness of the metal sheet and the resonant frequency and amplitude

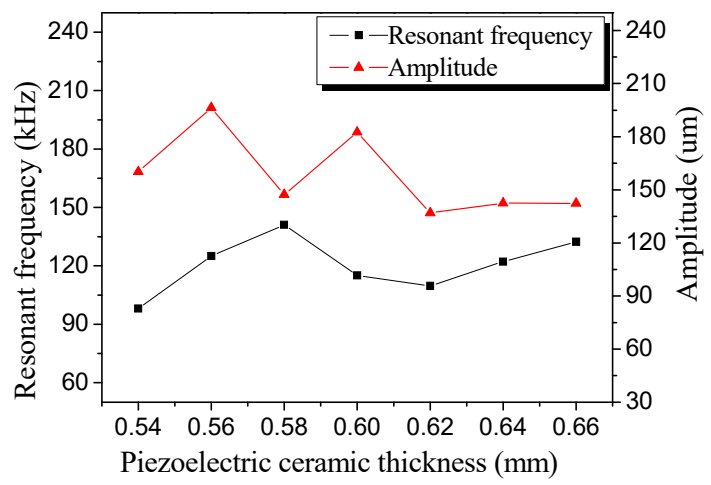

(d) The relationship between the Piezoelectric Ceramic Thickness and Resonant Frequency and Amplitude

Fig. 6. Relationship between atomization sheet with different parameters and resonance frequency and center point displacement 
It can be seen from Fig. 6 that the size and thickness of the inner and outer diameters of the piezoelectric ceramic ring have a significant influence on the resonance frequency, and within a certain range, the outer diameter is inversely proportional to the resonance frequency. Similarly, in different structural parameters, although peaks appear at some frequencies, by looking at the vibration cloud map corresponding to the frequency, the frequency is not available, indicating that the frequency cannot be used as a design reference [10], as shown in Figure 6-b. When the outer diameter is changed to $7.4 \mathrm{~mm}$, the resonance frequency is $149.87 \mathrm{kHz}$, and the maximum amplitude is 201.6um. However, by looking at the vibration cloud image as an unusable vibration mode, the frequency cannot be used as a design reference value. Therefore, in the design of the piezoelectric ceramic ultrasonic atomization sheet, a reasonable setting of various parameters is required to ensure the maximum amplitude at the resonance frequency, thereby achieving an optimal atomization effect.

\section{Conclusion}

The multi-order vibration mode of piezoelectric ceramic ultrasonic atomizing sheet can be obtained by modal analysis of ANSYS software, and a reasonable vibration mode can be selected, and the frequency-displacement response curve can be obtained by combining harmonic response analysis to determine atomization. The resonant frequency of the slice.

The relationship between response peak and frequency can be obtained by harmonic response analysis. The larger the vibration displacement at the center point, the higher the amount of fog and the better the atomization effect. According to the different size of the atomizer, the response peak condition can be reached under the harmonic response condition, and the main size of the atomizer can be optimized.

The inner and outer diameters and thickness of the piezoelectric ceramic ring have a significant influence on the resonant frequency, and within a certain range, the outer diameter is inversely proportional to the resonant frequency. In the design process of the atomized sheet, it is necessary to undergo multiple simulation analysis to find out the reasonable structural parameters and ensure the service life and atomization effect of the atomized sheet.

\section{Funding}

This research is supported by the Key Research and Development Program of Shanxi Province (201803D221028-2) and by the Natural Science Foundation of Shanxi Province (201701D121127).

\section{References}

1. F.X. Li, G.B. Li. Simulation Analysis of Radial Vibration of Axial Polarized Piezoelectric Ceramics. Coal Technology. 35(2), 285-286 (2016)
2. P. Du, N. Jiang, B. Song. Study on Frequency Characteristics and Matching of Ultrasonic Transducer. Electron Sound Technology, 40(1), 4145 (2016)

3. W. Chen, J. Yang. Analysis of Radial Vibration Mode of Piezoelectric Disc Oscillator Based on ANSYS. Computer Simulation, 26(3), 318-321 (2009)

4. L. Lin. Finite Element Simulation Analysis of Piezoelectric Ceramic Materials Based on ANSYS. Science and Technology Information, 36, 39-40 (2009)

5. X.L. Song. Research on mesh-type ultrasonic atomizer based on piezoelectric drive. Nanjing University of Aeronautics and Astronautics (2017)

6. J.M. Gao, Y.P. Lu, C.j. Liu, et al. Development and spray test of low frequency ultrasonic atomizing nozzle for micro-exponential oscillator. Transactions of the Chinese Society of Agricultural Engineering, 30(4), 40-46 (2014)

7. B.K. HAN, C SUN, H. Lu. Transient Characteristics Analysis of Pure Piezoelectric Vibrators Based on ANSYS. Micro motor, 40(4), 35-37 (2012)

8. H.Z. Ma. Research on Piezoelectric Effect and Piezoelectric Materials. Science and Technology Information, 30, 119-119 (2010)

9. F. Jiang, J.H. Zhang. Experimental research on ultrasonic atomization performance. National Symposium on Piezoelectric and Acoustic Theory and Device Applications, 1 ,150-153 (2014)

10. W. Chen, H.Y. Li. Design of a Novel Vibrating Mesh Atomizer. Mechanics \& Electronics, 2, 3-7 (2013) 